\title{
Towards Joint Routing and Spread-Spectrum Channelization in Cognitive Ad Hoc Networks
}

\author{
Kanke Gao and Lei Ding \\ Department of Electrical Engineering \\ The State University of New York at Buffalo \\ kgao, leiding@buffalo.edu
}

\begin{abstract}
We consider a spread-spectrum channelization based secondary network that operates cognitively to coexist with primary users. A new framework for cross-layer design that incorporates adaptation between spectrum-spreading channelization and routing is proposed. Joint power and spreading code allocation in conjunction of the back-pressure routing is developed to optimize the throughput over the whole network. The preliminary simulation results demonstrate significant performance gains compared to random spreading code assignment algorithm.
\end{abstract}

\section{Categories and Subject Descriptors}

C.2.1 [Computer Systems Organization]: Network Architecture and Design-Wireless communication

\section{General Terms}

Algorithms, design

\section{Keywords}

Spread-spectrum channelization, cognitive radio, power allocation, routing

\section{INTRODUCTION}

Cognitive radio networks $[1,2]$ have emerged as a promising technology to improve the utilization efficiency of the existing radio spectrum. Most cognitive radio studies attempt to find a single unused band which can be opportunistically used by secondary users, under the assumption that the primary users of the band are known a priori, and hence this knowledge may be incorporated into detecting if the band is occupied by the known signal pattern. In contrast, we propose a new spread-spectrum management paradigm, in which

Permission to make digital or hard copies of all or part of this work for personal or classroom use is granted without fee provided that copies are not made or distributed for profit or commercial advantage and that copies bear this notice and the full citation on the first page. To copy otherwise, to republish, to post on servers or to redistribute to lists, requires prior specific permission and/or a fee.

ACM CoNEXT 2010, November 30 - December 3, 2010, Philadelphia, USA.

Copyright 2010 ACM 978-1-4503-0468-9/10/11... \$10.00. waveforms are designed to occupy the entire available spectrum without generating harmful interference to active primary users. In this way, the secondary users share the licensed spectrum with the primary users to achieve frequency reuse. At the same time, the dynamic nature of the spectrum environment calls for the development of code-aware routing algorithm. Unfortunately, most spreading code designs do not provide interactions for higher layers to optimally adapt to underlying channel conditions.

The lack of established infrastructure and the wireless channel dynamics impose an unprecedented set of challenges over spread-spectrum-based cognitive ad hoc networks. The optimal spectrum-spreading channelization may be different at each hop in a multi-hop path. Furthermore, as new secondary links are formed and others vanish, routing of traffic from one secondary node to another may frequently change. Therefore, controlling the interaction between routing and code design is of fundamental importance.

In this work, we explore a new framework for crosslayer design that captures the interdependencies between spread-spectrum channelization and routing. The optimization is carried out dynamically by all secondary transmitters to continuously adapt to the changing spectrum environments and traffic flows. Preliminary simulation results demonstrate significant performance gains compared to random code assignment.

\section{JOINT ROUTING AND CODE-DIVISION CHANNELIZATION DESIGN}

Denote $\mathcal{P U}$ and $\mathcal{S U}$ as primary and secondary user sets, respectively. Traffic flows of secondary nodes are, in general, carried over multi-hop routes. Let $r_{i j}$ represent the capacity of secondary user link $(i, j), \forall i, j \in$ $\mathcal{S U}$, defined as $r_{i j}=\log _{2}\left(1+\mathrm{SINR}_{i j}\right)$, where $\mathrm{SINR}_{i j}$ represents the SINR of the link, whose value depends on the spreading code design and power allocation under the dynamic spectrum environment.

Power and Spreading Code Allocation: We now consider the opportunity to establish secondary code-division link $(i, j)$ coexisting with primary and on- 
going secondary code-division links. Our objective is to find the transmission power, real-valued normalized spreading code of the secondary link $(i, j)$ that maximizes the pre-detection SINR under the constraints that SINR of primary and ongoing secondary links are all above the threshold for primary users and secondary users, respectively. The optimization task is, unfortunately, a non-convex NP-hard optimization problem. We first relaxed the problem to a semidefinite program using the commutative property of trace and dropping the rank constraint. Then, we propose to use a primaldual interior-point method to solve this semidefinite program within the error $\epsilon$ in $O\left(L^{4} \log 1 / \epsilon\right)$ time, where $L$ is the length of the spreading code. If the rank-1 matrix with eigenvalue, eigenvector $\lambda_{1}, \mathbf{v}_{1}$ is, luckily, returned, then we have our secondary link design with code $\mathbf{v}_{1}$ and transmission power $\lambda_{1}$. Otherwise, using the randomized method in semidefinite programming literature, we can obtain a finite sequence of feasible solutions. To improve the "best" random solution, we use an iterative linearization procedure in which an iterative linearized polynomial-cost convex optimizer is developed with much appealing amplitude/code-vector design solutions after a few iterations.

Routing: Let the traffic demands consist of a set $\mathcal{S}$, and each session $s \in \mathcal{S}$ is characterized by a fixed sourcedestination node pair. Each node maintains a separate queue for each session $s$ for which it is either a source or an intermediate relay. At time slot $t$, define $Q_{i}^{s}(t)$ as the number of queued packets of session $s$ waiting for transmission at secondary user $i$. Note that, for the sake of simplicity, we will drop all time dependencies in the following. For each candidate next hop $j$, node $i$ estimates the maximum achievable link capacity $r_{i j}$ by optimizing the power and spreading code as described earlier. Schedule session $s^{*}$ with next hop $j^{*}$ such that

$$
\left(s^{*}, j^{*}\right)=\arg \max \left(r_{i j} \cdot\left(Q_{i}^{s_{i j}^{*}}-Q_{j}^{s_{i j}^{*}}\right)\right),
$$

where $s_{i j}^{*}=\arg \max _{s}\left\{Q_{i}^{s}-Q_{j}^{s}\right\}$, is the session with maximum differential backlog on link $(i, j)$. Hence, routing is performed in such a way that lightly backlogged queues with more spectrum resource receive most of the traffic. The objective function (1) is defined based on the principle of dynamic back-pressure, first introduced in [3]. It can be proven [4] that a control strategy that jointly assigns resources at the physical/link layers and routes to maximize the weighted sum of differential backlogs (with weights given by the achievable data rates on the link) is throughput-optimal, in the sense that it is able to keep all queues finite for any level of offered traffic within the network capacity region.

\section{PRELIMINARY RESULTS}

In this section, we compare the performance of the proposed solution with the random code assignment (RCA) scheme where routing is based on differential

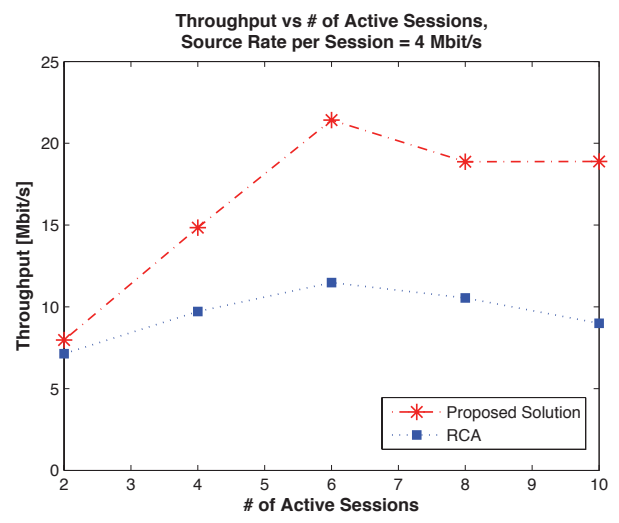

Figure 1: Throughput vs Time for Different Number of Active Sessions.

backlog (as in Section 2.2) with randomly generated spreading codes and fixed transmit power. The spreading code length is set to be 16 . A grid topology of 49 secondary nodes and 14 active primary links is deployed in $6000 \times 6000 m^{2}$ area. We initiate CBR traffic sessions between randomly selected but disjoint source-destination pairs. We vary the number of sessions injected into the network and plot the network throughput (sum of individual session throughput) in Fig. 1. With more active sessions, our proposed solution outperforms RCA since they use the best among possible spreading code and power allocations with routes adaptively. The preliminary results demonstrate significant performance gains in terms of network throughput.

\section{CONCLUSIONS AND FUTURE WORK}

The methodology presented in this paper is currently under study, to be refined and improved. We are currently testing it against other baseline solutions, and studying the effect of increasing the number of users and traffic load on the network performance in terms of throughput and delay.

\section{REFERENCES}

[1] I. F. Akyildiz,W. Lee, and K. Chowdhury, "CRAHNs: Cognitive Radio Ad Hoc Networks," Ad Hoc Networks Journal (Elsevier), vol. 7, pp. 810-836, Jul. 2009.

[2] J. Mitola, "Cognitive Radio Architecture Evolution," Proc. of the IEEE, vol. 4, pp. 626-641, Apr. 2009.

[3] L. Tassiulas and A. Ephremides, "Stability Properties of Constrained Queueing Systems and Scheduling Policies for Maximum Throughput in Multihop Radio Networks," IEEE Trans. on Auto. Contr., vol. 37, pp. 1936-1948, Jan. 1992.

[4] L. Georgiadis, M. J. Neely, and L. Tassiulas, "Resource Allocation and Cross-layer Control in Wireless Networks," Found. Trends Netw., 1(1):1-144, 2006. 